Article

\title{
Improving Waste Management System Efficiency and Mobility with Efficient Path MANET
}

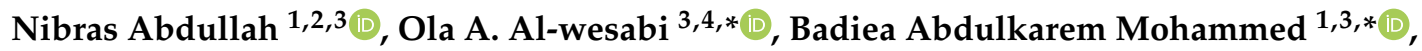 \\ Zeyad Ghaleb Al-Mekhlafi ${ }^{5}{ }^{\circ}$, Meshari Alazmi ${ }^{5}$, Mohammad Salih Alsaffar ${ }^{5}{ }^{\circ}$, Abdulaziz Salamah Aljaloud ${ }^{5} \oplus^{\circ}$, \\ Mahmoud Baklizi ${ }^{6}{ }^{(D)}$ and Putra Sumari ${ }^{4}$
}

1 Department of Computer Engineering, College of Computer Science and Engineering, University of Ha'il, Ha'il 81481, Saudi Arabia; neprasf@gmail.com

2 National Advanced IPv6 Centre, Universiti Sains Malaysia (USM), George Town 11800, Malaysia

3 Faculty of Computer Science and Engineering, Hodeidah University, Hodeidah P.O. Box 3114, Yemen

4 School of Computer Sciences, Universiti Sains Malaysia (USM), George Town 11800, Malaysia; putras@usm.my

5 Department of Information and Computer Science, College of Computer Science and Engineering, University of Ha'il, Ha'il 81481, Saudi Arabia; ziadgh2003@hotmail.com (Z.G.A.-M.); ms.alazmi@uoh.edu.sa (M.A.); m.alsaffar@uoh.edu.sa (M.S.A.); a.aljaloud@uoh.edu.sa (A.S.A.)

6 Department of Computer Information and Network Systems, The World Islamic Sciences and Education University, Amman 11947, Jordan; mbaklizi@wise.edu.jo

* Correspondence: ola.wosabi@gmail.com (O.A.A.-w.); b.alshaibani@uoh.edu.sa (B.A.M.); Tel.: +60-11-2847-4750 (O.A.A.-w.); +96-65-5855-2516 (B.A.M.)

check for updates

Citation: Abdullah, N.; Al-wesabi, O.A.; Mohammed, B.A.; Al-Mekhlafi, Z.G.; Alazmi, M.; Alsaffar, M.S.;

Aljaloud, A.S.; Baklizi, M.; Sumari, P. Improving Waste Management System Efficiency and Mobility with Efficient Path MANET. Appl. Sci. 2021, 11, 11039. https://doi.org/ 10.3390/app112211039

Academic Editor: Juan-Carlos Cano

Received: 15 October 2021

Accepted: 16 November 2021

Published: 22 November 2021

Publisher's Note: MDPI stays neutral with regard to jurisdictional claims in published maps and institutional affiliations.

Copyright: (c) 2021 by the authors. Licensee MDPI, Basel, Switzerland. This article is an open access article distributed under the terms and conditions of the Creative Commons Attribution (CC BY) license (https:/ / creativecommons.org/licenses/by/ $4.0 /)$.

\begin{abstract}
Waste Management System (WMS) is applied in smart cities and is supported by the Internet of Things (IoT). WMS involves several responsibilities, such as collection, disposal, and utilization of waste in relevant facilities. Efficient waste management has a considerable impact on the quality of life of citizens in smart cities. The interaction between wireless sensor networks and mobile ad-hoc networks (MANETs) with IoT provides excellent mobility for users, and reduces the deployment costs of networks. Data transfer via the intermediate connected devices must be maintained to shorten the distance between the devices. Intelligent routing algorithm is proposed to find efficient paths that comply with the WMS requirement constraints and avoid invalid paths that cause increased computation time. Chromosome intersection operation on genetic-based routing algorithm, intersection activation function, and intersection node table are proposed to avoid the similarity and redundancy of generated paths and keep the validity of the paths. This study improves the path finding, obtains good results, and increases the rate of efficient paths. Results show that the selected path is efficient in terms of distance, number of hops and number of iterations by using the proposed method. In addition, this proposed method outperforms DSR, which offers alternative paths with more efficiency.
\end{abstract}

Keywords: dynamic source routing (DSR); Internet of Things (IoT); mobile ad-hoc network (MANET); multi-constraints; routing; smart city; Waste Management System (WMS)

\section{Introduction}

In the current world of technology, various physical things can be used for smart cities and the facilitation of human work. Thus, the Internet of Things (IoT) is an innovative technology and a good solution, which allows the connection of physical things with the digital world through heterogeneous networks and communication technologies. The IoT in smart environments interacts with wireless sensor networks (WSN) and mobile ad hoc networks (MANET), making it highly attractive to users and economically successful.

The interaction between WSNs and MANETs with IoT allows the creation of new MANET-IoT systems and IT-based networks. These systems provide excellent mobility for users and reduce deployment costs of networks. MANETs are self-configuring networks of 
mobile routers connected via a wireless link. A MANET is the most promising and rapidly growing technology, and it has become a very popular research topic in recent years.

Smart cities result from the development of media transmission systems, which have expanded over the most recent 10 years through solid incorporation of networking advancements and data frameworks. In addition, the employments of portable specialised gadgets in daily life have been expanding. These gadgets include cell phones, business PCs, individual advanced gadgets with radios, and other computerized gadgets.

As a result of network revolution, different infrastructures of mobile and immovable systems coexist, and Internet applications have seen substantial growth. Even if these networks have wired or wireless connections, they must maintain data transfer via intermediate devices/nodes to shorten the distance between senders and receivers [1-4]. A wireless network enables users to be mobile and to save on infrastructure costs. The devices/nodes on ad hoc wireless networks are individual end user devices, and the devices can transmit data to one another $[5,6]$.

IoT devices are a part of a smart city with important mathematical capabilities, which are converted to smart objects used in monitoring the environment and collecting information about the environment in the city. The most critical problems facing smart cities today include waste management problems.

Waste Management System (WMS) is a typical example of a service provided by smart cities supported by IoT. Waste management involves different responsibilities, such as the collection, disposal, and utilization of waste in relevant facilities. Efficient waste management has a considerable impact on the quality of life of citizens. The reason is that waste disposal has a clear connection with negative impacts on the environment and, thus, on citizens health. Organic waste accounts for the majority of the wastes for disposal activities. Two factors are considered the main reasons for this problem: high urban growth rate and rapid population increase. Smart services can be the front line for this information on every aspect of human activity. WMS includes not only on-site waste collection but also waste disposal and uses in related facilities [7].

These services have different requirements, such as bandwidth and delay, for a network service provider to allow the applications to work properly [8-12].

However, other reasons, such as communication resources and routing algorithms, also contribute to the proper functioning of the applications. Routing is a methodical identification and decision making about sending data to specific destination nodes in accordance with their addresses. For example, the required action must be done by a certain prospective network member (worker/object device). Another example is a waste transportation system that needs an efficient algorithm to take the right way for waste collection at the right time. Its process consists of two steps: a path should be chosen based on service requirements, and each data packet should be sent along that path [13-18].

Open Shortest Path First (OSPF) and Routing Information Protocol (RIP) can provide different paths to the same destination by using multiple routing processes. Path selection is used to choose an available path to use. Thus, the router must consider some concepts, such as administrative distance (AD), because they influence the algorithm's path-selection process [19-24].

Thus, the default AD values (such as 110 for OSPF and 120 for RIP) are assigned to each routing algorithm. These values are set when the router receives the required routing configuration commands. Therefore, the route that minimizes the distance to the destination is selected and designated as the active route/path. The route selection process and algorithm used for each protocol is based on their requirements. For example, the routes are selected based on the lowest hop count criteria in RIP $[25,26]$.

The present study is focused on path selection optimization, which is dedicated to devising a well-rounded method that implements various constraints with the help of genetic algorithms (GA). At present, our best estimates of multipath routing algorithms indicate that they are all single-path constraint models, and they do not have a bearing on the relations amongst the constraints. MANET has many qualities, and some affect its 
performance; numerous factors affect its performance, and these elements correlate with the dynamic topology $[27,28]$. A large number of constraints must be considered to ensure that every constraint is accounted. An expeditious choice based on the route's effectiveness should be used [29-33]. Given the advances in recent genetic routing, pathfinding algorithms are currently most often used to identify a feasible path.

The rest of the paper is organized as follows: Material and methods are presented in Section 2. Section 3 explains the results and discussion of the experiments. Finally, Section 4 presents the conclusions.

\section{Related Work}

Different waste management models have been developed using certain IoT devices. Anghinolfi et al. [34] introduced a stage of municipal waste management using the collection of recycled waste data based on IoT innovation. Several waste management activities, such as waste collection, transport, and recycling, were also presented. However, an active optimization technique was proposed by Hannan et al. [35] for efficient waste recycling. An active selection that presents descriptions by identifying factors was developed. It compares the characteristics of wastes in a container on a day-to-day basis.

A specific monitoring system was proposed by Hannan et al. [35] to monitor garbage bins and trucks using Radio Frequency Identification (RFID) and information and communication technology. In addition to cameras, GPS is utilized in this system [35].

Garbage bins used for different types of waste are placed outside doors. Homogenous and heterogeneous trucks are used to collect waste [10,34,35].

In [36], bin collection is recorded to enhance the waste collection process. Figure 1 shows an example of small devices/gadgets with a web-based system that allows the integration of the IoT as a new generation technology.

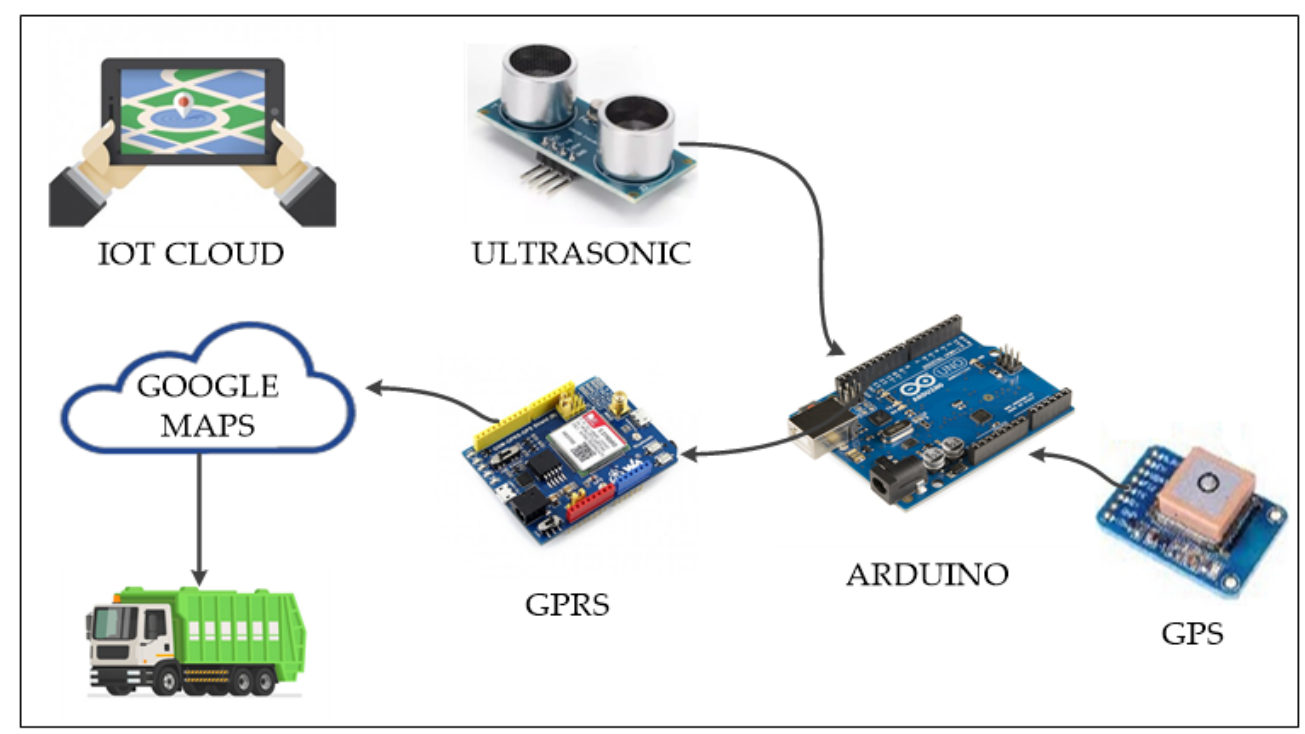

Figure 1. IoT based Waste Management System.

The architecture of the WMS consists of waste resource buildings to solve and manage various waste problems. Smart bins are used to improve waste collection and provide information for statistical analysis. Waste collection trucks are used to improve IoT-based Smart WMS in a smart city. The transportation of collected waste, workers, supervisors, and waste management centers are connected together, as interaction between WSNs and MANETs with IoT form the WMS. Figure 2 shows the proposed architecture. 


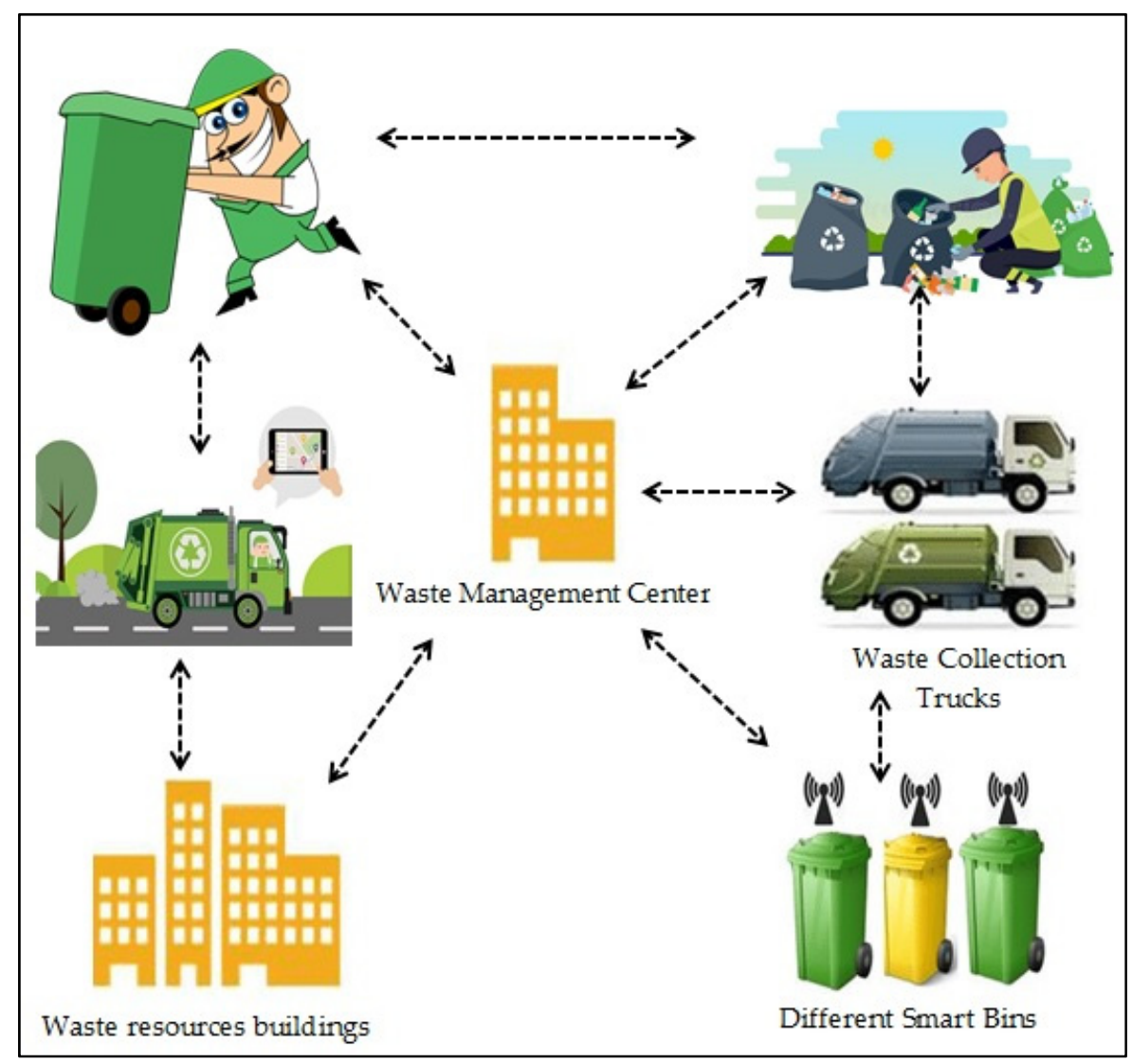

Figure 2. WMS architecture.

This section reviews the existing genetic-based routing algorithm. Considerable research on genetic-based routing algorithm field has been performed. The information from the related literature has been intensely reviewed to help motivate the methodology of the present study.

\section{1. $G A$}

GA is an evolutionary optimization strategy that draws inspiration from natural processes and is used in research on routing algorithms [37,38]. GA is a form of natural selection and evolutionary computing applied in global optimization [39-42]. GA has been demonstrated in theory and through experiments to be a reliable search method. GA-based routing algorithms are used in routing algorithms to find paths through networks [43].

As shown in Figure 3, the GA process starts by obtaining the population. In particular, it begins by randomly generating a population before evaluating individuals and finding solutions. This population is then tested using a fitness function that seeks to determine the function objective. After the evaluation, the best candidates for genetic operations, such as crossover and mutation, are selected to improve the next generation. Finally, the condition termination process is applied to see if the process should go on to the next round or be terminated.

Crossover operation, which is based on the GA structure, is the exchange of genes between pairs of selected individuals of chromosomes at specific points to generate a new generation of offspring with modified characteristics.

Chromosomes 1 and 2 are designated as parents or initial possible paths, whereas Chromosomes 3 and 4 are designated as new offspring, which may lead to the final solution. This process considers the suitable path, followed by the termination process or proceeding to the next iteration of the problem-solving process. 


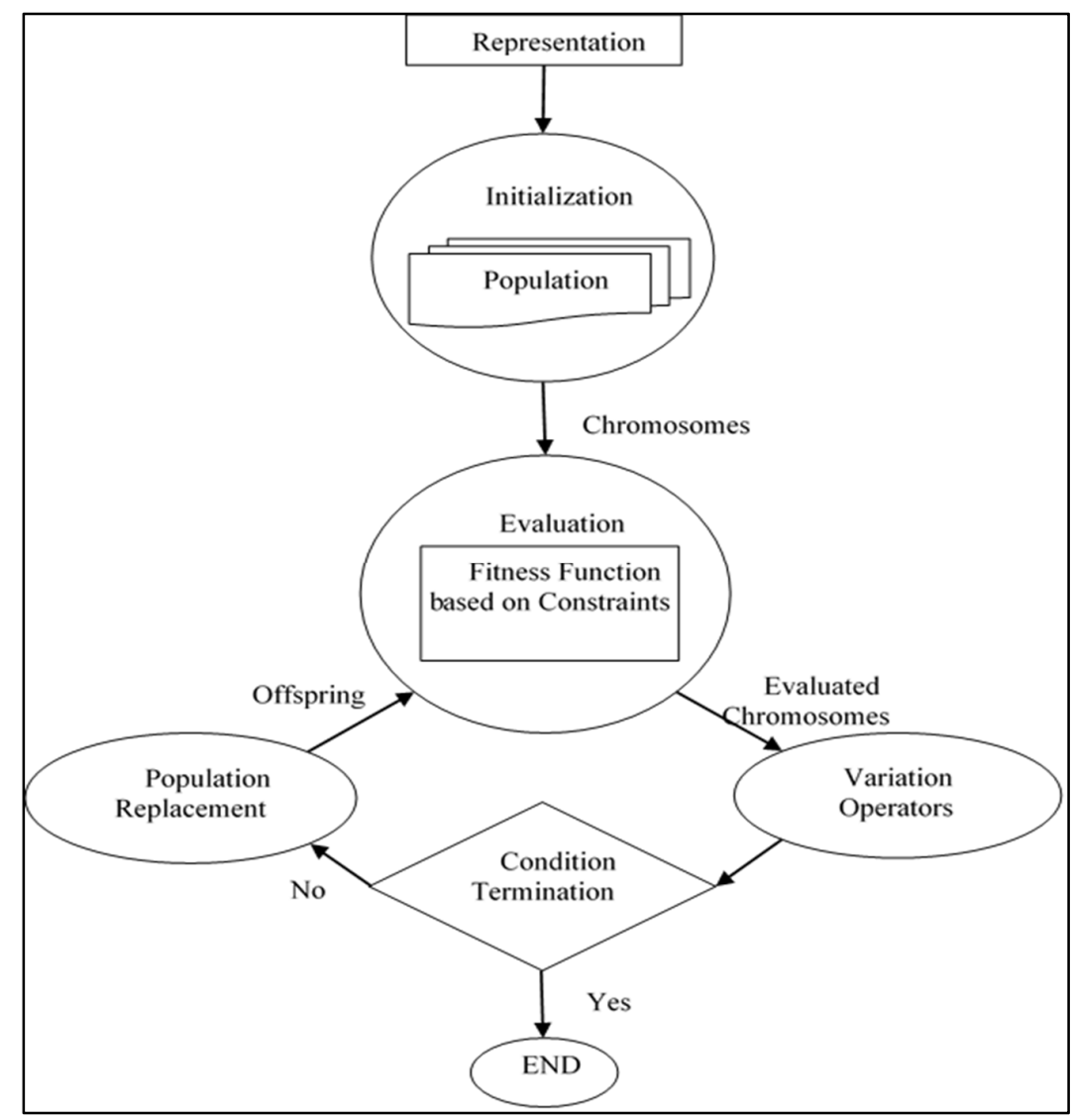

Figure 3. Designed GA flowchart.

The present study presents an intersection GA-based routing algorithm via a chromosome intersection method on the selected path during genetic processing to find the efficient paths that comply with the service requirement constraints. The influence of the intersection activation values in the proposed algorithm is also examined.

\subsection{GA-Based Routing Algorithm}

The representation of the routing problem in the GA structure is an important part of the features for the solutions that should be included. The result is that each chromosome represents a possible path from the source point to the destination point, making the chromosome's structure important.

GA is used in this study to solve the multiconstraint routing problem and find a efficient path that can comply with the service requirement for different network infrastructure types, such as WMS infrastructure. Routing selection based on multiconstraint is the most important problem that still needs to improve. When applying GAs to a routing problem, four important things must be addressed: Firstly, encoding or sampling the routing path; secondly, network representation; thirdly, population initialization; fourthly, the control of genetic operations on the path's representation chromosomes. The encoding routes affect population initialization and genetic operators, amongst other things. Furthermore, when a population is initialized, the size of the initial population and the strategy used to generate each individual must be considered.

Researchers at the University of California, Berkeley, developed genetic load balancing routing (GLBR) by presenting chromosomes in a network based on the sequence of nodes that form in the network and fixing the length of the chromosomes based on the number of nodes to ensure that the length of all chromosomes is equal to the number of nodes in the entire network [44]. Path selection is determined by the load balancing of the sent query packets. The first response path within that threshold value is chosen upon reaching 
a certain threshold value. In any other case, the GA recalculates to determine the best route to the destination. During the exchange of genes between a pair of chromosomes, the crossover operation forms invisible paths in the population solutions, which are called invisible paths. This phenomenon can occur when no relationship exists between the nodes at the end points of a crossover operation. Afterwards, the Dijkstra algorithm is used to mutate the chromosome, resulting in the mutation operation being carried out. This step increases the amount of time required for computation. The adaptive routing method based on GA with Quality of Service (QoS) (ARGAQ) is designed to overcome the looping problem in GLBR by employing another type of coding that is fixed and equal in length across all chromosomes [45]. The number of nodes in the chromosomes determines the length of the path that is followed. Checking for correct chromosome alignment is essential during the process of determining the initial path and after each genetic operation, which increases the amount of computation required. ARGAQ uses two constraints to improve the overall QoS [46].

In multiconstraint QoS unicast routing using GA, the initial population generated is based on the connectivity matrix, ensuring that all selected nodes are in the path from the source to the destination during the initial population-generating process. The proposed chromosome structure comprises several different elements, including duplicate genes, a linear list, genes, and nodes locus. These components aid in preventing routing loops and invisible paths. Active nodes are regions of each chromosome where the information is repeated constantly. The complex genetic operations performed on the chromosomes (such as the crossover and mutation operations) can improve and generate new offspring, but they require additional computation time to complete the task $[46,47]$.

Aside from the genetic operation, researchers used the same special genetic chromosome structure representing the network topology and the path that used marked signs inside the chromosomes as they did for the previous network topology and path. Thus, some methods only use the mutation operation, whereas other methods use the crossover and mutation operations. The fitness functions in the two constraints are also combined to form a single mixed constraint function.

The existing GA-based routing algorithm uses standard GA operations, namely, crossover and mutation operations, on the chromosomes, and may lead to invalid offspring, thereby increasing the computation and consumption time. Additionally, the newly generated chromosomes in every generation must be applied and verified. Thus, knowing the validity of genes in the chromosomes is essential when using the GA operations.

In the next section, the proposed method integrates specific operations to avoid invalid paths and find the efficient path.

\section{Intersection GA-Based Routing Algorithm}

This section focuses on the proposed intersection GA-based routing selection design.

\subsection{Initialisation}

The initialization stage presents the initial population as a research space to find the feasible path. The representation of network topology in a symmetric matrix, in addition to the intersection GA-based algorithm, is the proposed intersection node table presented in Table 1. The proposed intersection node table is used to generate the chromosomes and prevent invalid paths in the initial population. The proposed relation table is generated based on the connectivity matrix. All connected devices are presented as state nodes in the first row and neighbors for each node in the second row, which are considered the next hope for the current state, as shown in Table 1.

Table 1. Intersection node table.

\begin{tabular}{|c|c|c|c|c|c|c|c|c|c|c|c|c|c|c|c|c|c|c|c|c|c|c|c|}
\hline State & & 1 & & & 2 & & & & 3 & & & 4 & & & & 5 & & & 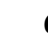 & & & 7 & \\
\hline $\begin{array}{l}\text { Next } \\
\text { state }\end{array}$ & 2 & 3 & 5 & 1 & 4 & 5 & 1 & 5 & 6 & 7 & 2 & 5 & 8 & 1 & 2 & 3 & 4 & 8 & 3 & 7 & 3 & 6 & 8 \\
\hline
\end{tabular}


According to the intersection node table, the selected state in the first gen in the chromosome presents the source node as the starting point towards the destination point on the last gene of the chromosome. A random selection of intermediate nodes is made from the neighboring active nodes connected to the current state node, starting with the source node and progressing until the destination. Each chromosome in the initial population is generated using the same generation process according to the number of chromosomes, which is identified by the population size.

\subsection{Intersection Activation Function Procedure}

Based on the constraints, the population chromosomes are evaluated using the intersection activation function. Each chromosome presents an available path. The calculated intersection activation value defines the efficient path to be selected. The constraint parameter in this work is cost, which is considered as distance and delay. Equation (1) is used to calculate the weight given to each path and is written as

$$
w_{i}(p)=\sum_{(u \rightarrow v) \in p}\left(w_{i}(u \rightarrow v) \leq K_{i} \text { for all } i=1, \ldots, l\right),
$$

where $l$ is the total number of constraints, $p$ is the path, $K_{i}$ is the maximum constraint value, and $w_{i}$ is the path weights.

Based on Equation (1), the intersection activation value is calculated as presented in Equation (2):

$$
\mathrm{F}(\mathrm{P})=\operatorname{Max}\left(w_{1}(p), w_{2}(p), w_{3}(p), \ldots, w_{l}(p)\right) .
$$

\subsection{Individual Path Selection and Population Generation}

From the initial population, which is considered as the expected paths that can be used for the service applied for, one path from the source node to the destination node is presented by each chromosome in the population on a single linkage map. All the chromosomes are evaluated with Equation (2), which calculates the intersection activation value for each path. Then, the chromosomes are ranked according to the calculated activation value.

Chromosomes with low activation values are chosen as the best candidates. In the worst-case scenario, chromosomes with values that are either outside the range of the constraints or very close to the boundary values are considered problematic. Removing undesirable chromosomes and replacing them with new chromosomes becomes possible using the proposed chromosome intersection method. This finding means that the most efficient path based on the constraint values is found on the best chromosomes, which have the most favorable values. The top-ranked chromosome is recombined with one of the next highest-ranked chromosomes through floor-off division. The next highest-ranked chromosomes are chosen based on the chromosome's intersection point and presented in Equation (3).

$$
\text { Intersection point }=\text { floor_off (Chromosome_length/2). }
$$

\subsection{Chromosome Intersection Method}

The chromosome intersection method is a recombination process that generates new chromosomes by exchanging and combining the genes amongst the chromosomes. When the standard crossover is used, the index of the randomly selected point is used to exchange the genes on a pair of selected chromosomes. The proposed chromosome intersection method is used based on the information of the selected intersection point to find the exchanging point through the chromosomes and obtain new chromosome generation. The chromosome intersection method is applied to the chromosomes with the highest ranking. The intersection point is calculated according to the length of the chromosome using Equation (3). 
The operation selects the next chromosome as a partner depending on the right components of the calculated intersection point to reach the target node. This action guarantees the connectivity and validity between the selected pair of chromosomes.

The following are the steps involved in the chromosome intersection method:

- According to the intersection activation value calculated from the evaluation stage by Equation (2), the available rank of the chromosomes for the current generation is calculated;

- The intersection activation value, which is the most important factor in the combination process of a pair of chromosomes, is used to select the best chromosome from the population. Then, the best chromosome from the population is selected;

- The intersection point in the selected chromosome is identified using Equation (3), and the content value of the intersection point is used to determine where the intersection point is located. A new set of valid chromosomes is created by exchanging the chromosomes for their corresponding genes;

- After the intersection point is selected, a new intersection point is selected from the next highest ranked chromosome if the selected point is not found either in the chromosome currently in use or in the next highest-ranked chromosome;

- The selected chromosomes are recombined by exchanging the gene parts between them in accordance with the content of the intersection point that presents the node ID in the available path, which is determined by the intersection point's content;

- The newly combined chromosomes are replaced with the chromosomes with a low level of activation value;

- Afterwards, the condition is terminated after the intersection activation function evaluation has been completed.

Chromosome intersection method is used instead of the two GA operations, namely, crossover and mutation, which are applied in the index of the crossover point. These methods do not guarantee the chromosome part connection. However, the content of the intersection point guarantees the connection between the nodes, reducing the number of iterations, and avoiding the loop that performs search space reduction.

Figure 4 shows the crossover point for standard crossover, and Figure 5 shows the intersection point for chromosome intersection samples. The chromosome intersection method influences the processing time as follows:

1. Available paths are presented by the newly generated chromosomes that result from the recombination operation;

2. Verifying the generated chromosomes is not needed;

3. This operation is applied instead of two genetic operations.

Standard Crossover

Original chromosomes (parents)

\begin{tabular}{|c|c|c|c|c|c|c|c|}
\hline Crossover point index 3: & 1 & 2 & & & 5 & 6 & \\
\hline Chromosome_1 & 2 & 3 & 4 & 6 & 7 & 8 & 9 \\
\hline \multicolumn{3}{|l|}{ Crossover point index 3: } & & \multirow[b]{2}{*}{4} & \multirow[b]{2}{*}{7} & \multirow[b]{2}{*}{8} & \\
\hline Chromosome 2 & 2 & 5 & 6 & & & & 9 \\
\hline After standard crossover & & & & & & & \\
\hline Chromosome 1 & 2 & 3 & 6 & 4 & 7 & 8 & 9 \\
\hline Chromosome 2 & 2 & 5 & 4 & 6 & 7 & 8 & \\
\hline
\end{tabular}

Figure 4. Standard crossover samples. 
Chromosomes intersection

Original chromosomes (parents)

\begin{tabular}{|c|c|c|c|c|c|c|c|}
\hline Intersection-poin & 1 & & & & & & \\
\hline Chromosome 1 & 2 & 3 & 4 & & 1 & 8 & 9 \\
\hline Intersection-poin & inir & & & & & & \\
\hline Chromosome 2 & 2 & 5 & 6 & & 7 & 8 & 9 \\
\hline After Chromosol & $\overline{\mathrm{sec}}$ & & & & & & \\
\hline Chromosome_1 & 2 & 3 & 4 & 7 & 8 & 9 & \\
\hline Chromosome 2 & 2 & 5 & 6 & 4 & 7 & 8 & 9 \\
\hline
\end{tabular}

Figure 5. Chromosome intersection samples.

\section{Experiment Results and Discussion}

In this section, three experiments are presented. The experiments were performed on network topologies with four different sizes. The parameters for each scenario were determined based on the randomness of the network's behavior. The network sizes in these experiments were 20,30, 40, and 50 nodes. The first experiment discovered the routes from the source node to all other nodes as destinations and evaluated the performance of the chromosome intersection method in the proposed algorithm.

The second experiment examined the intersection GA-based routing algorithm to find the efficient path based on the multiconstraint's values from the source node to the specific destination node.

The third experiment compared the proposed intersection GA-based routing selection and dynamic source routing (DSR). DSR is one of the common reactive routing algorithms in MANET. Thus, it is considered the best in the ad hoc network field.

The simulation experiment was performed using ns- 2 according to the simulation setup shown in Table 2.

Table 2. Experiment setup.

\begin{tabular}{ll}
\hline Network Devices Nodes & 50 \\
\hline Routing protocol & DSR \\
\hline Network type & MANET \\
\hline X dimension of the topography & 1000 \\
\hline Y dimension of the topography & 1000 \\
\hline
\end{tabular}

\subsection{Experiment 1}

This experiment intends to discover the routes from the source node to all other nodes as destinations. This experiment evaluated the performance of the chromosome intersection method and the intersection activation function's integration in the proposed algorithm. Various network sizes [20, 30, 40, and 50] of devices/nodes were used in two scenarios. In the first scenario, the possible path was selected without any constraint priority. In the second scenario, the possible paths were selected based on the priority of the constraints. The system should consider and be concerned with both constraints which have the same priority.

The scenarios described above were investigated to determine the most efficient path from the source node 0 to the other nodes in the same topology with a maximum cost value of 100 , which denoted the distance between the devices. 


\subsubsection{Scenario 1}

In this scenario, the performance of the intersection GA-based routing algorithm was analyzed to find the compliance path. The proposed algorithm selected the most efficient path with the minimum intersection activation value based on the calculated intersection activation value. Then, the rest of the acceptable paths were considered alternative paths. Figure 6 shows the destinations and the intersection activation values for the available paths towards each destination for different numbers of devices/nodes in the network. Figure $6 \mathrm{a}-\mathrm{d}$ show that the number of available paths obtained based on the chromosome intersection operation value was increased when the number of nodes was increased for the same destination node. Thus, the algorithm supports a great number of devices and users in the system. However, the available paths based on the ranked intersection activation value (RIAV) present the most efficient path without the constraint priority.

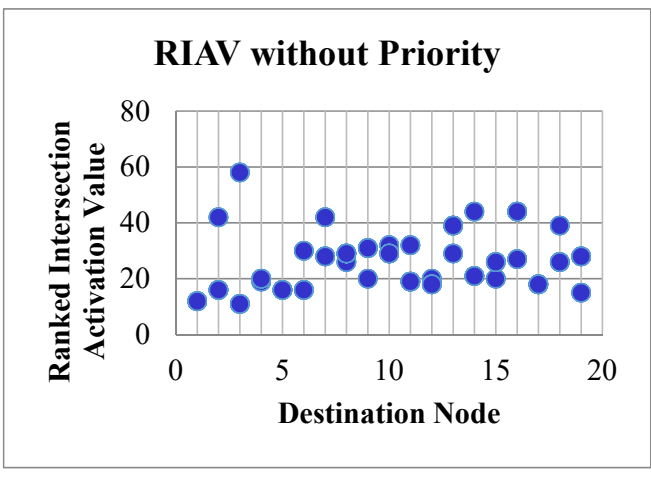

(a)

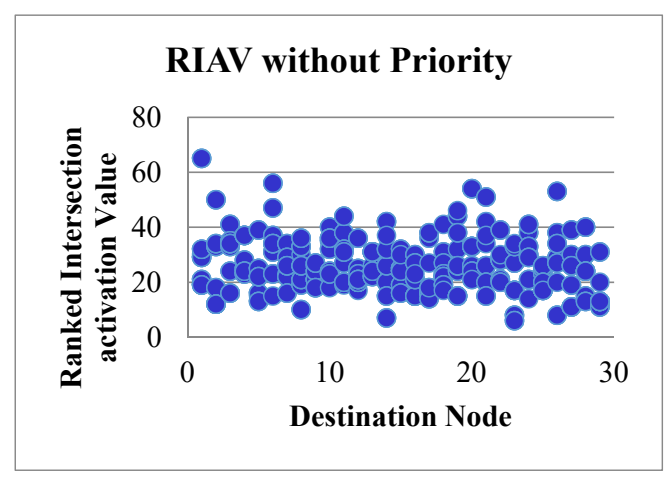

(b)

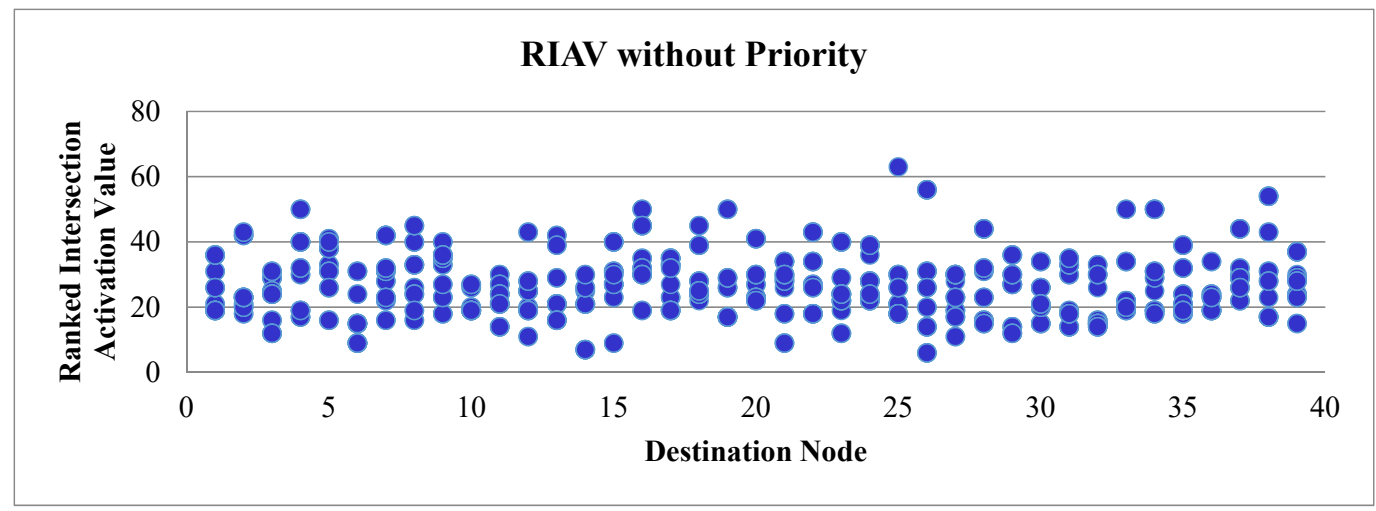

(c)

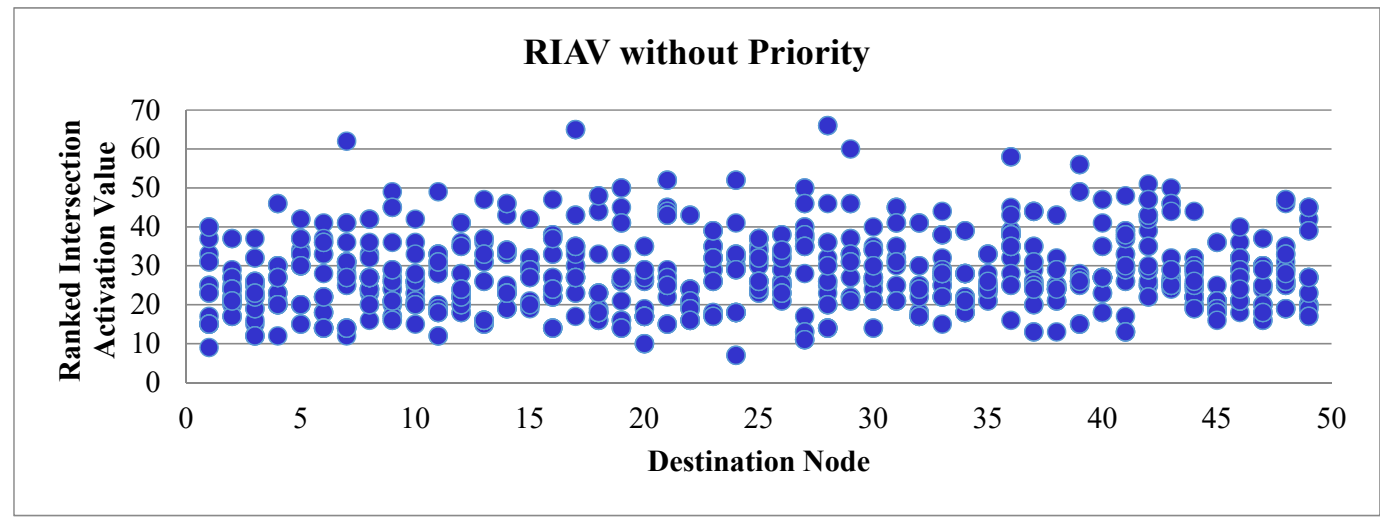

(d)

Figure 6. Available paths towards each destination. (a) Available paths with 20 nodes; (b) Available paths with 30 nodes; (c) available paths with 40 nodes; (d) available paths with 50 nodes. 
Figure 7 shows that the cost constraint values for all available paths in the same networks for the maximum cost value in the scenario are less than 70, and the majority of the available paths are less than $50 \%$ from the permitted cost value. The objective of this study is to minimize the cost of the selected path. This method reduced the processing of routing selection based on the proposed intersection GA-based routing selection. Compared with the conventional GA-based routing algorithms, the proposed algorithm uses chromosome intersection operation, which can control and avoid invalid paths, thereby improving results and providing an efficient path.

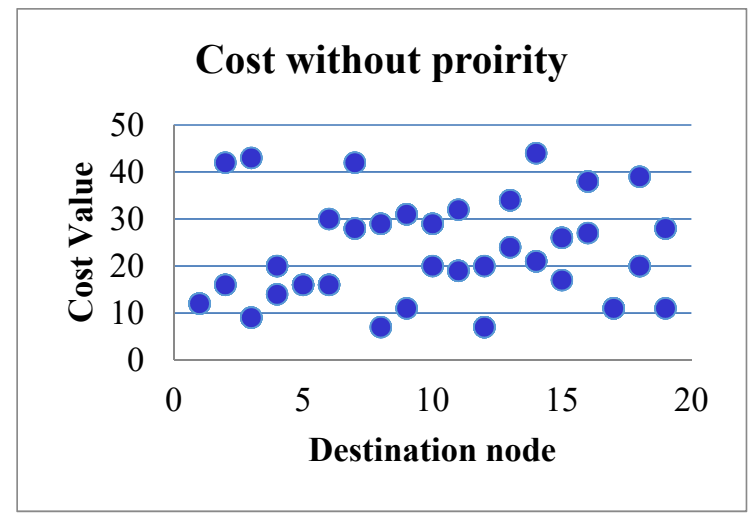

(a)

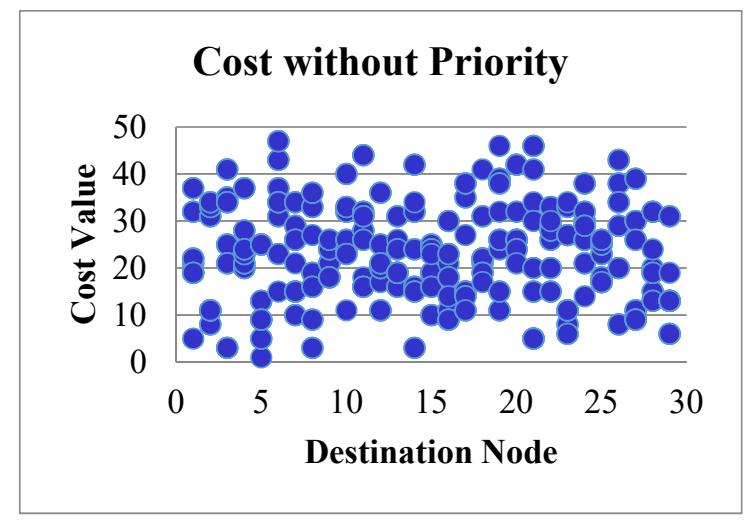

(b)

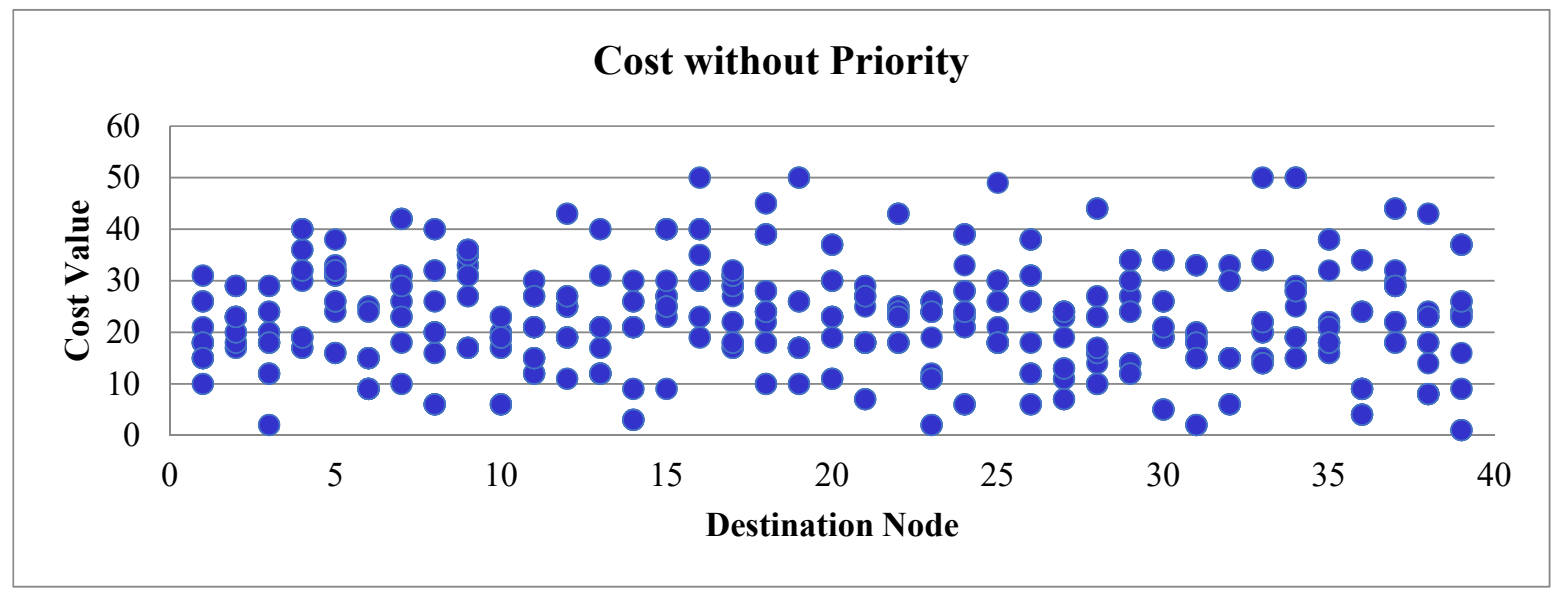

(c)

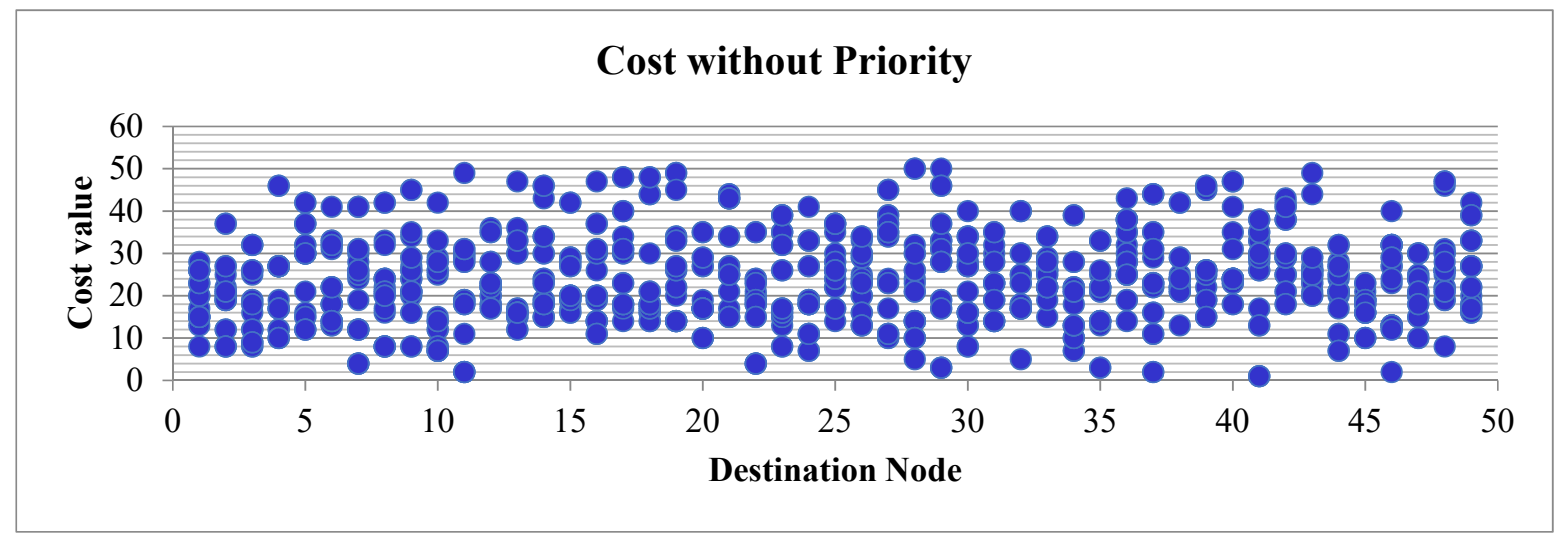

(d)

Figure 7. Available paths in the same networks. (a) Available paths with 20 nodes; (b) available paths with 30 nodes; (c) available paths with 40 nodes; (d) available paths with 50 nodes. 


\subsubsection{Scenario 2}

The results of this scenario demonstrated the effectiveness of the proposed intersection GA-based routing algorithm when subjected to priority constraints. The results of the same experiments showed that the selected paths from the source node 0 to other nodes in the same network for different network topology sizes are shown in the results for the same experiments. The available paths from node 0 to other nodes are shown in Figure 8. The calculated intersection activation values were presented based on constraint priority.

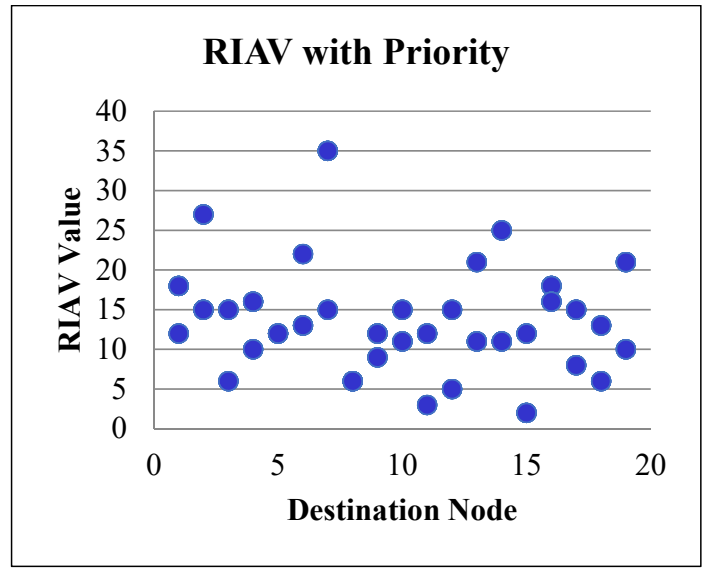

(a)

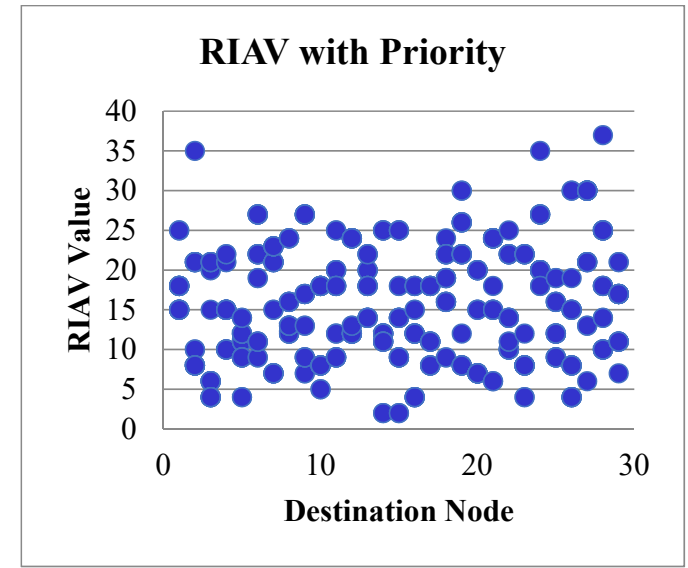

(b)

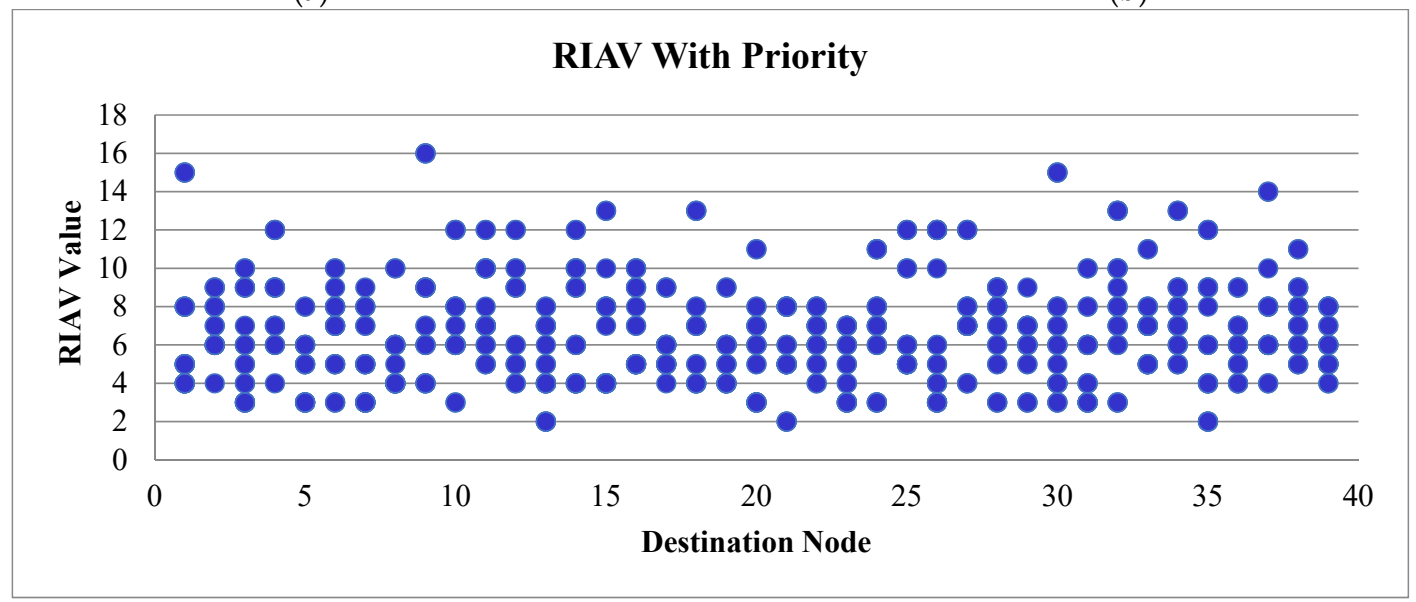

(c)

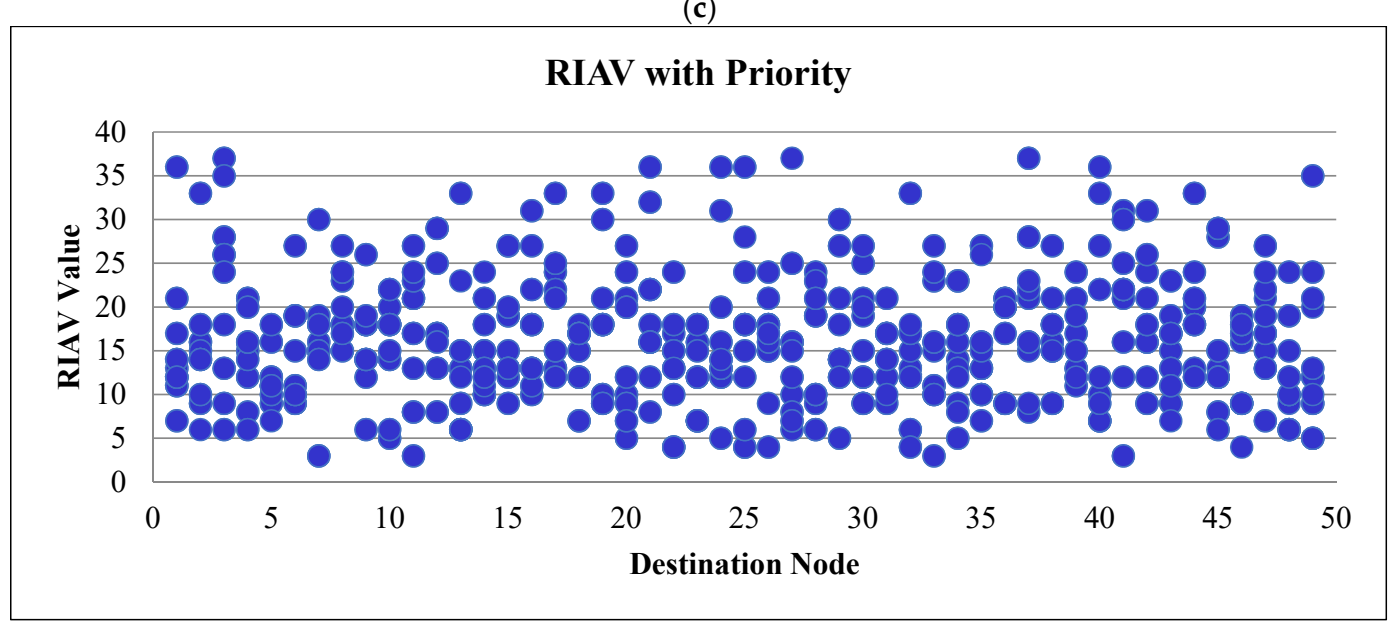

(d)

Figure 8. Calculated intersection activation values based on the constraint priority. (a) Available paths with 20 nodes; (b) available paths with 30 nodes; (c) available paths with 40 nodes; (d) available paths with 50 nodes. 
Similar to Scenario 1, Scenario 2 exhibited an increase in the number of available paths when the number of nodes was increased in the network. However, the calculated intersection activation values in Scenario 2 were less than those in Scenario 1. The efficient path has a low activation value. Thus, all the available paths in Scenario 2 are more efficient than those in Scenario 1.

The constraint values that can produce efficient paths with priority are shown in Figure 9. With respect to different network sizes, these values represent the bare minimum cost of all available paths to all network nodes starting from the source node 0 for each network size.

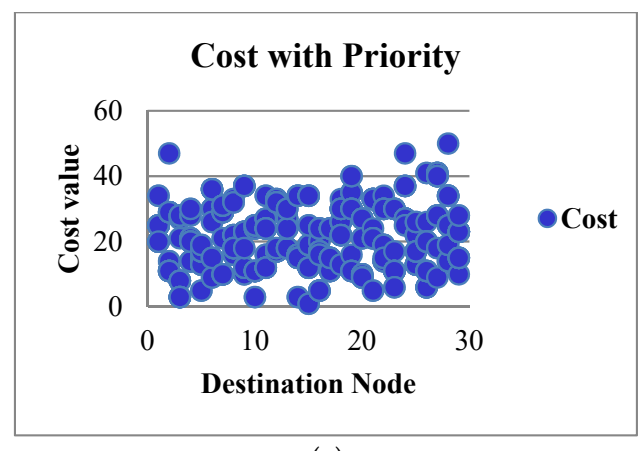

(a)

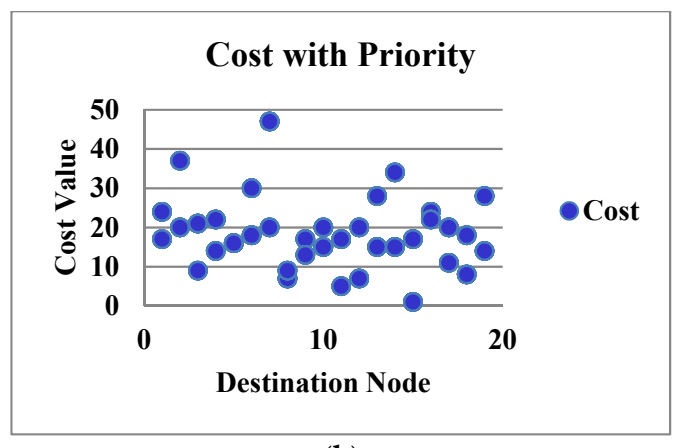

(b)

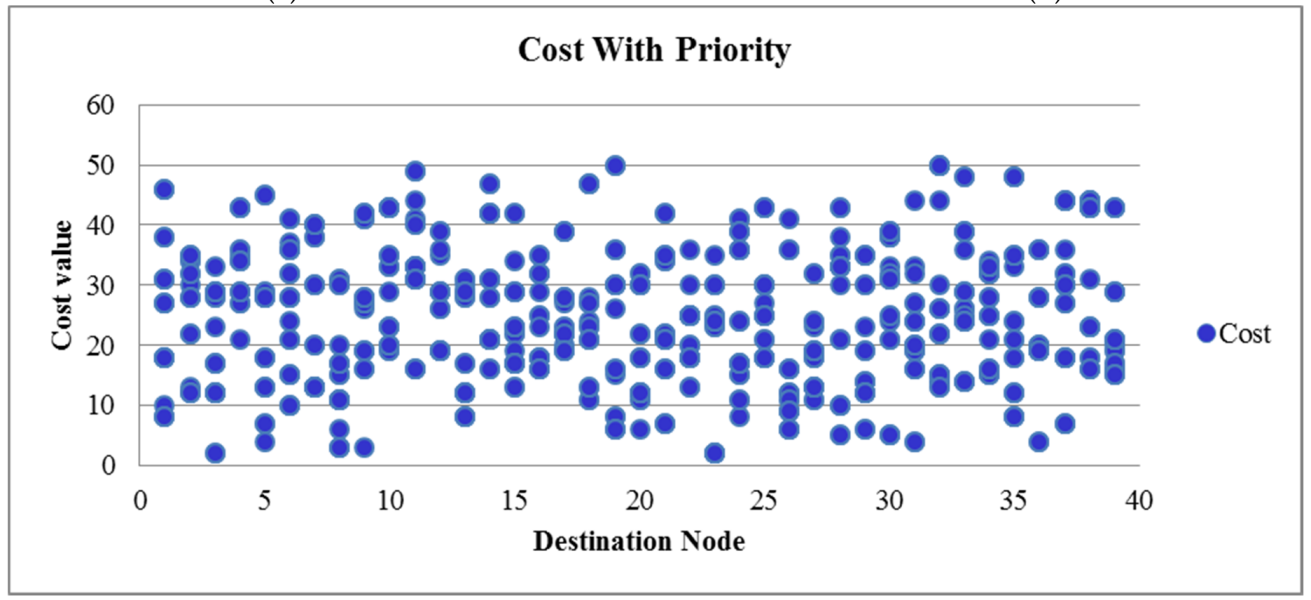

(c)

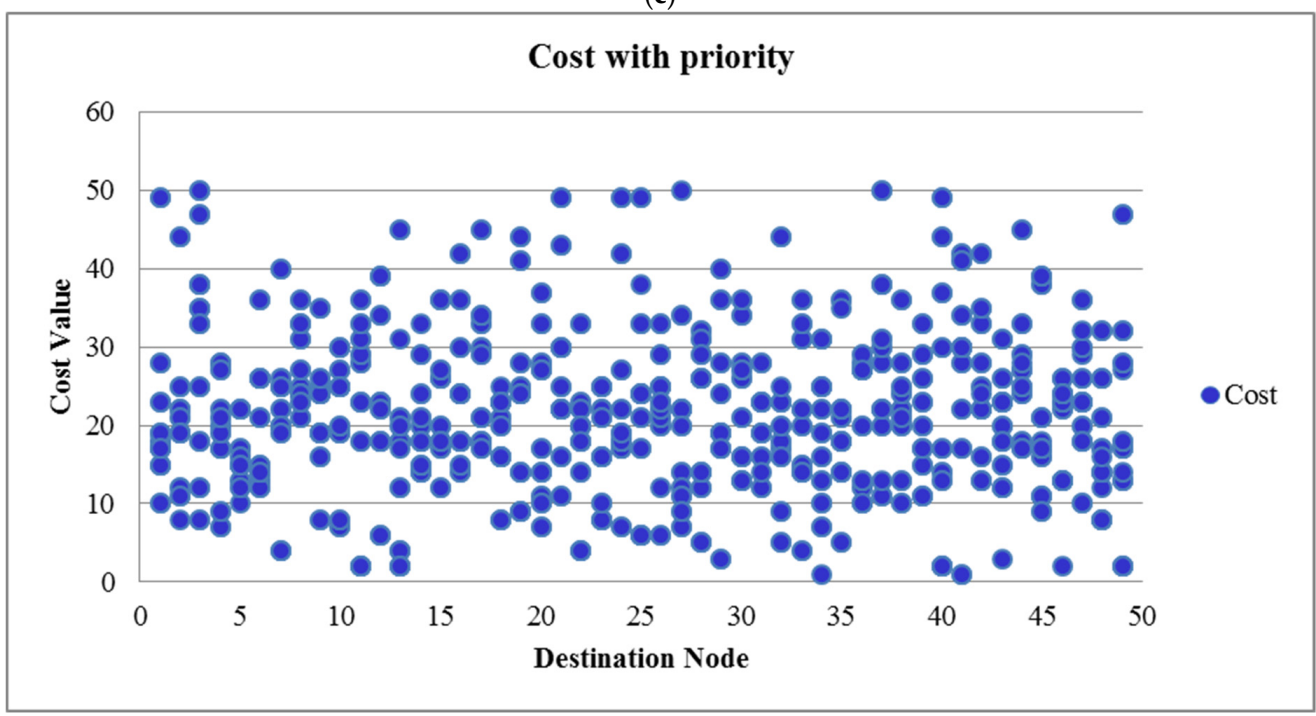

(d)

Figure 9. Constraint values that can produce efficient paths with priority. (a) Available paths with 20 nodes; (b) available paths with 30 nodes; (c) available paths with 40 nodes; (d) available paths with 50 nodes. 
The two scenarios in experiment 1 showed that the number of available paths to each destination node is increased when the network size (the number of devices) is increased and may offer a good quality connection amongst the neighbors. The selected paths using intersection GA-based routing algorithm without priority have 50\% of the maximum constraint value. However, the efficiency of path selection in Scenario 2 with intersection GA-based routing algorithm with priority is increased by $20 \%$ compared with that in Scenario 1. Therefore, the efficiency of the selected path is increased as the number of nodes is increased, and the algorithm performs effectively. Moreover, the findings indicated that the intersection GA-based routing algorithm is reliable in finding the most efficient path with other alternative efficient paths based on multiple constraints when chromosome intersection is employed.

\subsection{Experiment 2}

In this experiment, the intersection GA-based routing algorithm was applied to find the efficient path based on the multiconstraint's values from source node 0 to a specific destination node. Table 3 presents the constraints and the calculated intersection activation value for the top five chromosomes from the generation during four rounds of iterations. Each chromosome presents a single path from the source node 0 to the destination node.

Table 3. Constraints of available paths.

\begin{tabular}{ccccc}
\hline Iteration & Path No. & Constraint One & Constraint Two & Intersection Activation Value \\
\hline \multirow{4}{*}{1} & Path 0 & 11 & 41 & 41 \\
& Path 1 & 43 & 40 & 43 \\
& Path 2 & 50 & 61 & 61 \\
& Path 3 & 83 & 61 & 83 \\
& Path 4 & 96 & 91 & 96 \\
\hline \multirow{4}{*}{2} & Path 0 & 11 & 41 & 41 \\
& Path 1 & 43 & 40 & 43 \\
& Path 2 & 50 & 61 & 61 \\
& Path 3 & 43 & 40 & 43 \\
& Path 4 & 43 & 40 & 43 \\
\hline \multirow{4}{*}{3} & Path 0 & 11 & 41 & 41 \\
& Path 1 & 43 & 40 & 43 \\
& Path 2 & 50 & 61 & 61 \\
& Path 3 & 25 & 65 & 65 \\
\hline \multirow{6}{*}{4} & Path 4 & 83 & 61 & 41 \\
& Path 0 & 11 & 41 & 43 \\
& Path 1 & 43 & 40 & 61 \\
& Path 2 & 50 & 61 & 65 \\
& Path 3 & 25 & 65 & 83 \\
\hline
\end{tabular}

As shown in Table 3, the intersection activation values are affected by the chromosome intersection operation during the processing, which continues for other iterations. The chromosome intersection operation recombined the chromosomes to generate new chromosomes and replaced the worst-case chromosomes in the population. The presented results are the top five chromosomes representing the most efficient paths. The calculated intersection activation value considered the maximum constraint values, which must be acceptable and in compliance with the system requirements. The 4 th and 5 th chromosomes representing paths 3 and 4 were updated in iteration 3 . They remain the same in iteration 4 .

Intersection GA-based routing algorithm selects the paths that have the lowest fitness values based on the intersection activation value results. Therefore, the new offspring replaces the chromosomes that are detrimental to the population. The good chromosomes are kept with the updated values during the iterations that show the ability of the proposed method to find the efficiency of the path. 
Furthermore, the quality of the paths based on the two constraints is improved because of the results presented. Figures 10 and 11 show the values of constraints one and two, respectively, and the feasible paths for the same scenario. Path 0 has the minimum value amongst the available paths, that is, $22 \%$ from the maximum acceptable value by decreasing $78 \%$ in constraint one and $59 \%$ in constraint two. Consequently, path 0 is the efficient selected path. Constraint one values of path 1 during iteration 1 are the same and are more efficient than constraint two values by decreasing $60 \%$ of the maximum acceptable value of constraint two. Path 2 has the same constraint one values during the second iteration with the maximum acceptable value, whereas constraint two values present $40 \%$ of the maximum value. Constraint one values for paths 3 and 4 are improved by $69.8 \%$ and $13 \%$, respectively, from the initial value, making path 3 an acceptable path and in compliance with the constraint's restrictions. Additionally, constraint two of path 3 is increased by $6.5 \%$ during iteration 3 , and that of path 4 is improved by $32 \%$ from the initial values. Hence, paths $0,1,2$, and 3 are the acceptable paths. According to Figure 11, the fitness value for each path is determined by the constraint applied to the path. The intersection activation values calculated remain constant because the constraint values of paths 0,1 , and 2 remain constant throughout the four iterations. However, the intersection activation values of paths 3 and 4 are improved by $21.6 \%$ and $13 \%$, respectively.

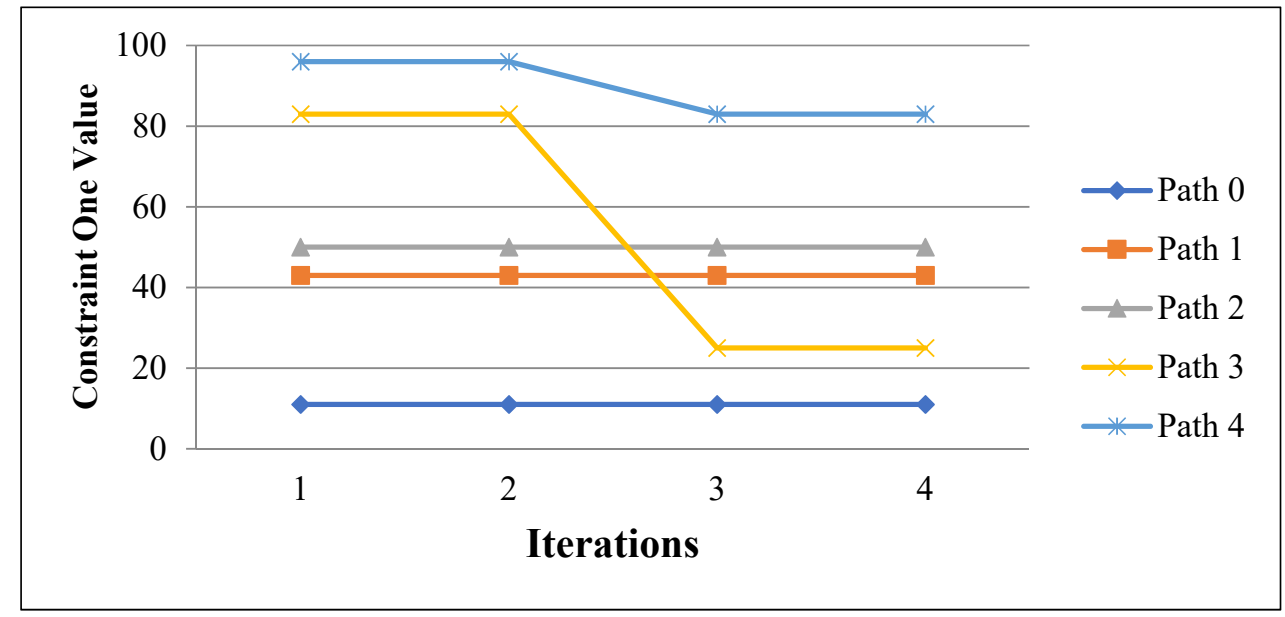

Figure 10. Constraint one of feasible paths.

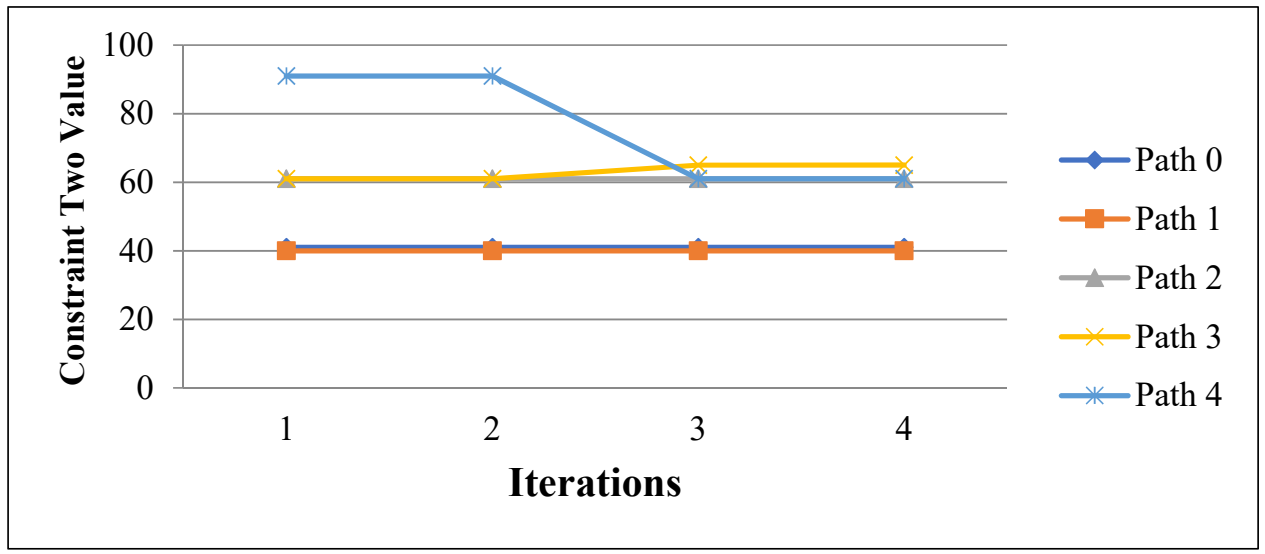

Figure 11. Constraint two of feasible paths.

The improved ranking of the individuals produces the acceptable paths at the top of the population list. The minimum intersection activation value is the most efficient amongst the available paths. The objectives of lowering the cost must be considered to identify the feasible paths, even though the cost value that can be used is limited to 100 . The 
results shown in the previous graphs demonstrated that the presented routing algorithm, which is based on an intersection GA-based routing algorithm operation, can achieve high overall performance for all the feasible paths. The graphs showed that the majority of the cost values for all available paths to the same destination are less than the maximum limit of the cost value, resulting in better results and more efficient path selection than the maximum cost value.

However, the selected paths using intersection GA-based routing algorithm without priority have $50 \%$ of the maximum constraint value. Compared with scenarios without priority, intersection GA-based routing algorithm with priority increases the efficiency of path selection by $20 \%$.

Thus, the efficiency of the selected path increases as the number of nodes increases, and the performance of the algorithm improves as the number of nodes increases. The evaluation experiment outcomes indicated that the proposed intersection GA-based routing algorithm is trustworthy enough to find an efficient path based on multiple constraints.

The results of these experiments confirmed that the efficiency of the available paths based on the two constraints can be improved considerably by employing the proposed intersection GA-based routing algorithm during the iterations. The chromosome intersection operation is one of the most important operations in the GA process. On the basis of genetic-based routing algorithm, this study investigated the effect of chromosome intersection method. The chromosome intersection method depends on the content of the intersection point, whereas the standard crossover operation depends on the location of the crossover point.

\subsection{Experiment 3}

In this experiment, the performance of the intersection GA-based routing algorithm was compared with that of DSR. In this experiment, the source node was fixed at point $(x=0 ; y=0)$, and the destination node was fixed at position $(x=950 ; y=950)$ to find the efficient path between the two end points.

Figure 12 shows the intermediate node number of the proposed method and DSR for the selected path, as a result of this experiment. Figure 13 presents the travel distance taken by the selected path between the two end points for the same network. The proposed method selected alternative paths in addition to the efficient selected path, whereas DSR selected only one path. The result indicated that DSR selects a single path between the two end points with 13 intermediate nodes. This number is the same as that of the intermediate nodes of the efficient selected path in the proposed method. Given that the chromosome intersection method was used, the proposed method selected the efficient path in terms of distance. The distance of the efficient path selected by the proposed method is less than that by DSR. However, the transmission time is decreased, and the connection is strengthened.

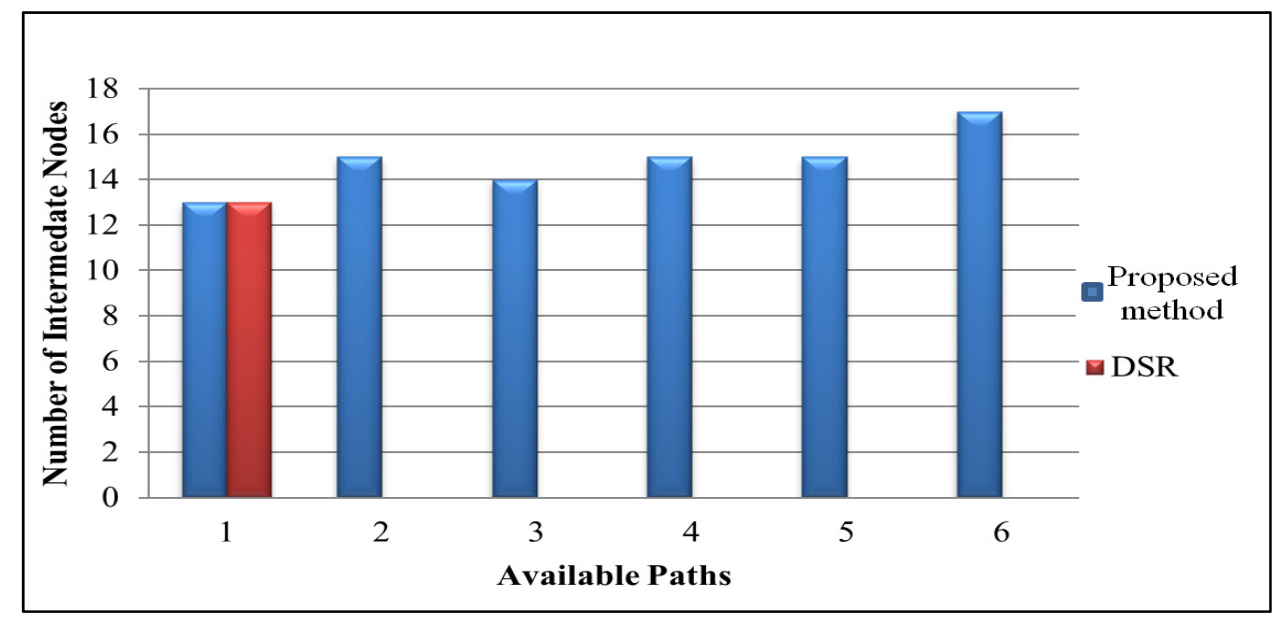

Figure 12. Intermediate node counts. 


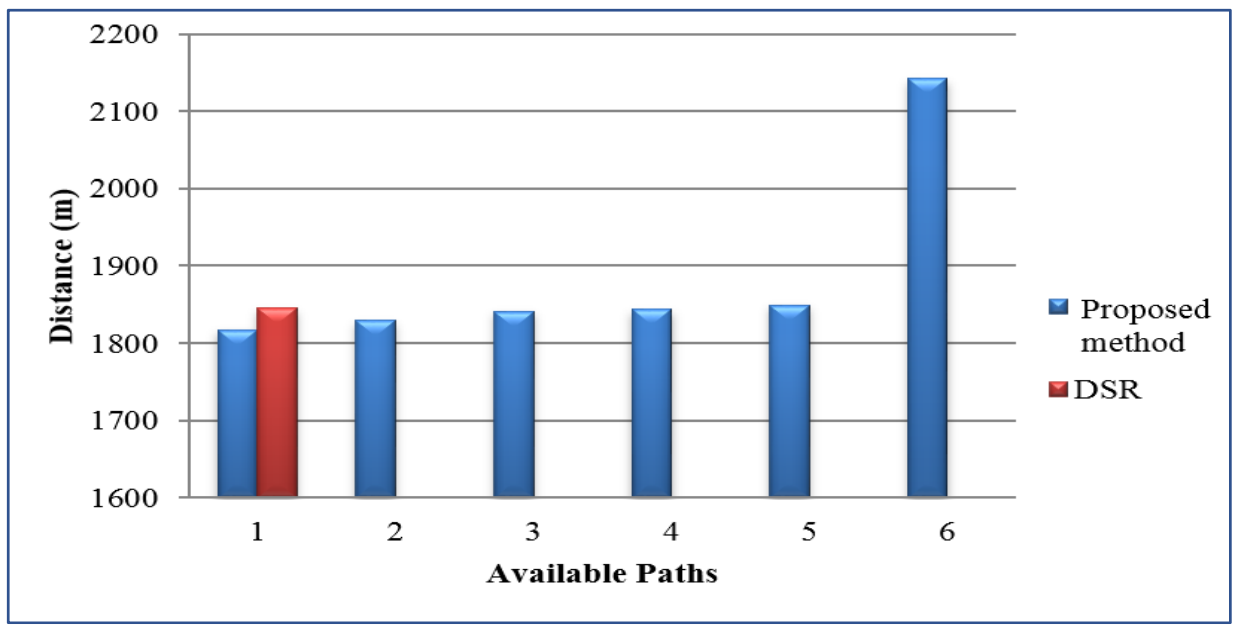

Figure 13. Selected path.

We can understand from the experiments above the most important effects of the chromosome's intersection method on the processing time as follows:

- The newly generated chromosomes, which results from the recombination operation, display the currently available pathways;

- Checking the chromosomes that have been generated is unnecessary;

- This operation is used in place of two genetic operations that would otherwise be used.

The intersection activation function is the most important function in evaluating the chromosomes' performance. The chromosomes were ranked based on their activation function values to identify the most efficient path amongst the available paths in the genome. An abnormally high intersection activation value was considered the worst possible chromosome and should not be passed on to the next generation. Thus, the good chromosomes continue to replicate throughout the processing process until the termination condition is satisfied. These experiments confirmed that the efficiency of the available paths based on the two constraints is improved using the proposed method during the iterations.

This research contributes to design the intersection GA-based routing algorithm to improve WMS efficiency and mobility with efficient path MANET. Its goal is to find an efficient path among the available paths. The genetic structure was designed to present the route from the source to the destination and the information required for finding the efficient path. The genetic structure includes the chromosomes that present the path, and the priority of constraints needed for obtaining the efficient path. In addition, the use of the chromosome intersection method helped to minimize the processing time. The scenarios that applied to find the efficient path based on two constraints, show the improvement in the quality of chromosomes during the iterations, which is affected by the priority of constraints. The minimum RIAV of the chromosomes produces the efficient path. The good chromosomes continue during the processing unless it generates a new and better chromosome, then it will take its place based on the RIAV. In addition, the proposed method offers better results for different network sizes and different destination nodes. It has been shown that the priority has an effect on the constraints values of the available paths. The constraints values are far from the maximum bounded values, which present efficient paths in the scenario that concerns priority constraints more than other scenarios that do not concern the priority of constraints. Moreover, the performance comparison of the proposed method to DSR showed that the proposed method provides an efficient path in terms of the number of intermediate nodes and distance. In addition, it provides alternative paths while DSR only offers one path. 


\section{Conclusions}

Waste management issues are the most challenging problems in smart cities. An IoT-based Waste Management System is provided by smart cities. WMS involves several responsibilities, such as collection, disposal, and utilization of waste in relevant facilities. Efficient waste management has a considerable impact on the quality of life of citizens. The interaction between wireless sensor networks and mobile ad hoc networks with IoT provides excellent mobility for users and reduces the deployment costs of networks. This study considers the population and urban growth by using a different number of devices/nodes that ease communication among system entities, such as waste source areas, waste collection trucks, and waste management centers. Different infrastructures of mobile and immovable systems coexist, and Internet applications have seen substantial growth So, it should maintain data transfer via intermediate devices/nodes to shorten the distance between senders and receivers. An intelligent routing algorithm is proposed to find the efficient paths and reduce the computation time of route selection. An intersection GAbased routing algorithm is proposed to find the efficient paths and reduce the computation time of route selection. Intersection activation function applies the intersection operation step, which is the important step of the proposed method. The experimental results of compliance path selection in MANET show the optimized path selection using the proposed method compared with the DSR. This finding confirms that the proposed method reduces the processing time and avoids the invalid solutions that may be generated during the iterations. The results show that the upper chromosomes representing the compliance paths are close to one another and within the acceptable values. In addition, the use of priority constraints required by the system improves outcomes at considerable constraints by choosing the best solution and using the remaining chromosomes as an alternative pathway. This process allows the proposed method to override the existing methods.

Author Contributions: Conceptualization, N.A. and O.A.A.-w.; methodology, N.A. and O.A.A.-w.; writing—original draft preparation, N.A., O.A.A.-w. and M.B.; writing-review and editing, Z.G.A.-M., M.A., M.S.A. and A.S.A.; supervision, P.S.; project administration, B.A.M. All authors have read and agreed to the published version of the manuscript.

Funding: This research has been funded by the Scientific Research Deanship at the University of Ha'il, Saudi Arabia, through project number RG-20 044.

Institutional Review Board Statement: Not applicable.

Informed Consent Statement: Not applicable.

Data Availability Statement: Not applicable.

Acknowledgments: We would like to acknowledge the Scientific Research Deanship at the University of Ha'il, Saudi Arabia, for funding this research.

Conflicts of Interest: The authors declare no conflict of interest.

\section{References}

1. Wang, W.; Wang, J.; Wang, M.; Wang, B.; Zhang, W. A Realistic Mobility Model with Irregular Obstacle Constraints for Mobile Ad Hoc Networks. Wirel. Netw. 2017, 25, 487-506. [CrossRef]

2. Raza, A.; Kumar, P.; Ahmed, A.; Khan, U.A. Comprehensive survey of routing protocols for wireless body area networks (WBANs). In Industrial Internet of Things and Cyber-Physical Systems: Transforming the Conventional to Digital; Kumar, P., Ponnusamy, V., Jain, V., Eds.; IGI Global: Hershey, PA, USA, 2020; pp. 145-178. [CrossRef]

3. Goyal, A.; Sharma, V.K.; Kumar, S.; Kumar, K. Modified Local Link Failure Recovery Multicast Routing Protocol for MANET. J. Inf. Optim. Sci. 2020, 41, 669-677. [CrossRef]

4. Trivedi, R.; Khanpara, P. Robust and secure routing protocols for MANET-based internet of things systems-A survey. In Emergence of Cyber Physical System and IoT in Smart Automation and Robotics; Singh, K.K., Nayyar, A., Tanwar, S., Abouhawwash, M., Eds.; Springer: Cham, Switzerland, 2021; pp. 175-188. [CrossRef]

5. Jose, M.R.; Singh, J.A.P. Security Challenges for Routing Protocols in Mobile Ad Hoc Network: A systematic review and Open research issues. Int. J. Electron. Secur. Digit. Forensics 2021, 13, 268-297. [CrossRef] 
6. Sarao, P. Ad Hoc On-Demand Multipath Distance Vector Based Routing in Ad-Hoc Networks. Wirel. Pers. Commun. 2020, 114, 2933-2953. [CrossRef]

7. Abdullah, N.; Alwesabi, O.A.; Abdullah, R. IoT-Based Smart Waste Management System in a Smart City. In International Conference of Reliable Information and Communication Technology; Saeed, F., Gazem, N., Mohammed, F., Busalim, A., Eds.; Springer: Cham, Switzerland, 2018; Volume 843, pp. 364-371. [CrossRef]

8. Nidhya, R.; Kumar, M.; Ravi, R.V.; Deepak, V. Enhanced Route Selection (ERS) Algorithm for IoT Enabled Smart Waste Management System. Environ. Technol. Innov. 2020, 20, 101116. [CrossRef]

9. Jayakumar, N.; Joshi, D.P. Big Data \& Disruptive Computing Platforms Braced Internet of Things. In Facets E Trends Internet of Things, Smart Computing and Technology: A Roadmap Ahead, Studies in Systems, Decision and Control; Dey, N., Mahalle, P., Shafi, P., Kimabahune, V., Hassanien, A., Eds.; Springer: Cham, Switzerland, 2020; Volume 266, pp. 119-150. [CrossRef]

10. Rajkumar, M.N.; Abirami, P.; Kumar, V.V. Smart garbage collection monitoring systems. Int. J. Sci. Res. Comput. Sci. Eng. Inf. Technol. 2017, 2, 13-17.

11. Santos, J.; Wauters, T.; Volckaert, B.; De Turck, F. Towards Delay-Aware Container-Based Service Function Chaining in Fog Computing. In Proceedings of the NOMS IEEE/IFIP Network Operations and Management Symposium, Budapest, Hungary, 20-24 April 2020; IEEE: Piscataway, NJ, USA, 2020; pp. 1-9.

12. Kassab, W.; Darabkh, K.A. A-Z Survey of Internet of Things: Architectures, Protocols, Applications, Recent Advances, Future Directions and Recommendations. J. Netw. Comput. Appl. 2020, 163, 102663. [CrossRef]

13. Praveen, K.V.; Prathap, P.M.J. Energy Efficient Congestion Aware Resource Allocation and Routing Protocol for IoT Network Using Hybrid Optimization Techniques. Wirel. Pers. Commun. 2021, 117, 1187-1207. [CrossRef]

14. De Irigon, J.I.; Walter, F.; Springer, T. Evaluation of DTN Routing Algorithms. In Scheduled Public Transport Networks; Grieco, L.A., Boggia, G., Piro, G., Jararweh, Y., Campolo, C., Eds.; Springer: Cham, Switzerland, 2020; Volume 12338, pp. 37-52. [CrossRef]

15. Khalil, M.; Khalid, A.; Khan, F.U.; Shabbir, A. A Review of Routing Protocol Selection for Wireless Sensor Networks in Smart Cities. In Proceedings of the 2018 24th Asia-Pacific Conference on Communications (APCC), Ningbo, China, 12-14 November 2018; IEEE: Piscataway, NJ, USA, 2018; pp. 610-615. [CrossRef]

16. Martinez, I.; Hafid, A.S.; Jarray, A. Design, Resource Management, and Evaluation of Fog Computing Systems: A Survey. IEEE Internet Things J. 2021, 8, 2494-2516. [CrossRef]

17. Zrelli, A. Hardware, Software Platforms, Operating Systems and Routing Protocols for Internet of Things Applications. Wirel. Pers. Commun. 2021, 1-24. [CrossRef]

18. Reddy, K.S.; Yashoda, M.B. Intelligent Routing Protocols for Communication in Internet of Things: A Survey. J. Comput. Theor. Nanosci. 2018, 15, 3291-3296. [CrossRef]

19. Howser, G. Open Shortest Path First. In Computer Networks and the Internet; Springer: Cham, Switzerland, 2019; pp. 271-297. [CrossRef]

20. Valadas, R. OSpF and IS-IS: From Link State Routing Principles to Technologies, 1st ed.; CRC Press: Boca Raton, FL, USA, 2019.

21. Gember-Jacobson, A.; Akella, A.; Mahajan, R.; Liu, H.H. Automatically Repairing Network Control Planes Using an Abstract Representation. In Proceedings of the 26th Symposium on Operating Systems Principles, Shanghai, China, 28-31 October 2017; ACM: New York, NY, USA, 2017; pp. 359-373. [CrossRef]

22. Abhashkumar, A.; Gember-Jacobson, A.; Akella, A. Aed: Incrementally synthesizing policy-compliant and manageable configurations. In Proceedings of the 16th International Conference on emerging Networking EXperiments and Technologies, Barcelona, Spain, 1-4 December 2020; ACM Press: New York, NY, USA, 2020; pp. 482-495. [CrossRef]

23. Matqurbonov, D.; Nushtayeva, O.; Tojiyeva, F. Model and Algorithms for Distribution of Information Flows in Data Transmission Networks. In Proceedings of the 2019 International Conference on Information Science and Communications Technologies (ICISCT), Tashkent, Uzbekistan, 4-6 November 2019; IEEE: Piscataway, NJ, USA, 2019; pp. 1-4. [CrossRef]

24. Nevzorova, O.; Lemeshko, O.; Mersni, A.; Hailan, A.; Ali, A.S.; Harkusha, S. Improved Two-Level Method of Multicast Routing in MPLS-TE Network. In Proceedings of the 2019 IEEE 2nd Ukraine Conference on Electrical and Computer Engineering (UKRCON), Lviv, Ukraine, 2-6 July 2019; IEEE: Piscataway, NJ, USA, 2019; pp. 846-850. [CrossRef]

25. Prakash, S.W.; Deepalakshmi, P. Flow-Based Dynamic Load Balancing Algorithm for the Cloud Networks Using Software Defined Networks. Int. J. Cloud Comput. 2019, 8, 299-318. [CrossRef]

26. Ganie, A.G. Optimization of PSWAN in Terms of Cost and Bandwidth. Int. J. Inf. Technol. Bus. 2018, 1, 42-47. [CrossRef]

27. Panda, N.; Panda, P. A Secure and Energy Efficient Ant Routing Algorithm. Intell. Decis. Technol. 2020, 14, 325-336. [CrossRef]

28. Chengetanai, G.; Osunmakinde, I.O. Swarm Intelligence on MANET Routing Issues: An empirical study revealing latent research topics. Int. J. Mob. Netw. Des. Innov. 2019, 9, 119-133. [CrossRef]

29. Ujjwal; Thangaraj, J.; Kumar, R. Multi-Path Provisioning in Elastic Optical Network with Dynamic on-Request Optimal Defragmentation Strategy. Opt. Switch. Netw. 2021, 41, 100607. [CrossRef]

30. Zhao, N.; Long, X.; Wang, J. A Multi-Constraint Optimal Routing Algorithm in LEO Satellite Networks. Wirel. Netw. 2021, 1-12. [CrossRef]

31. Zhang, X.; Liu, Z.; Zhang, M.; Li, L. An experimental study on exact multi-constraint shortest path finding. In Databases Theory and Applications; Qiao, M., Vossen, G., Wang, S., Li, L., Eds.; Springer: Cham, Switzerland, 2021; Volume 12610, pp. 166-179.

32. Lai, X.; Li, J.; Chambers, J. Enhanced Center Constraint Weighted a Algorithm for Path Planning of Petrochemical Inspection Robot. J. Intell. Robot. Syst. 2021, 102, 1-15. [CrossRef] 
33. Garaaghaji, A.; Alfi, A. Robust Performance Rate Control to Enhance MANET Networks Routing Issue. J. Electr. Eng. Technol. 2019, 15, 477-486. [CrossRef]

34. Anghinolfi, D.; Paolucci, M.; Robba, M.; Taramasso, A.C. A Dynamic Optimization Model for Solid Waste Recycling. Waste Manag. 2013, 33, 287-296. [CrossRef]

35. Hannan, M.A.; Arebey, M.; Begum, R.; Basri, H. Radio Frequency Identification (RFID) and communication technologies for Solid Waste Bin and Truck Monitoring System. Waste Manag. 2011, 31, 2406-2413. [CrossRef] [PubMed]

36. Vicentini, F.; Giusti, A.; Rovetta, A.; Fan, X.; He, Q.; Zhu, M.; Liu, B. Sensorized Waste Collection Container for Content Estimation and Collection Optimization. Waste Manag. 2009, 29, 1467-1472. [CrossRef]

37. Cao, Y.; Wu, M. A Novel RPL Algorithm Based on Chaotic Genetic Algorithm. Sensors 2018, 18, 3647. [CrossRef]

38. Opoku-Amankwaah, A.; Ombuki-Berman, B. An Age Layered Population Structure Genetic Algorithm for the Multi-Depot Vehicle Problem. In Proceedings of the 2017 IEEE Symposium Series on Computational Intelligence (SSCI), Honolulu, HI, USA, 27 November-1 December 2017; IEEE: Piscataway, NJ, USA, 2017; pp. 1-8.

39. Cheng, C.-Y.; Lin, S.-W.; Pourhejazy, P.; Ying, K.-C.; Li, S.-F.; Liu, Y.-C. Greedy-Based Non-Dominated Sorting Genetic Algorithm III for Optimizing Single-Machine Scheduling Problem with Interfering Jobs. IEEE Access 2020, 8, 142543-142556. [CrossRef]

40. Zhu, N.; O'Connor, I. iMASKO: A Genetic Algorithm Based Optimization Framework for Wireless Sensor Networks. J. Sens. Actuator Netw. 2013, 2, 675-699. [CrossRef]

41. Ibrahim, A.O.; Shamsuddin, S.M.; Abraham, A.; Qasem, S.N. Adaptive Memetic Method of Multi-Objective Genetic Evolutionary Algorithm for Backpropagation Neural Network. Neural Comput. Appl. 2019, 31, 4945-4962. [CrossRef]

42. Liang, Y.; He, T.-P. Survey on Soft Computing. Soft Comput. 2020, 24, 761-770. [CrossRef]

43. Zheng, Z.; He, F.; Xiong, H. Routing Optimization of Time-Triggered Ethernet Based on Genetic Algorithm. In Proceedings of the 2020 AIAA/IEEE 39th Digital Avionics Systems Conference (DASC), San Antonio, TX, USA, 11-15 October 2020; IEEE: Piscataway, NJ, USA, 2020; pp. 1-8.

44. Shahhoseini, H.S.; Torkzadeh, S. Fast Converging Evolutionary Strategy for Multi-Constraint QoS Routing in Computer Networks Using New Decoding Mechanism. Comput. Inform. 2017, 36, 405-422. [CrossRef]

45. Torkzadeh, S.; Soltanizadeh, H.; Orouji, A.A. Multi-Constraint QoS Routing Using a Customized Lightweight Evolutionary Strategy. Soft Comput. 2018, 23, 693-706. [CrossRef]

46. Wang, X.; Zhang, J.; Huang, M.; Yang, S. A Green Intelligent Routing Algorithm Supporting Flexible QoS for Many-to-Many Multicast. Comput. Netw. 2017, 126, 229-245. [CrossRef]

47. Lu, T.; Chang, S.; Guo, W.; Huang, Q. Genetic Algorithm for Energy-Aware QoS Multicast Routing in MANETs. Computer 2017, 5 , 10-20. 